\title{
Meeting highest performance requirements for lowest price and mass for the M1 segment support unit for E-ELT.
}

\author{
Jan Nijenhuis, Roger Hamelinck, Ben Braam, TNO Science and Industry, Stieltjesweg 1, 2623 CK \\ Delft, The Netherlands.
}

M. Cayrel, ESO, Karl Schwartzschildstrasse 2, 85748 Garching bei München, Germany

\begin{abstract}
The largest optical telescope in the world will be the E-ELT. Its primary mirror will be $42 \mathrm{~m}$ in diameter. This mirror will consist of 984 hexagonal segments that are all individually supported. Each mirror will be controlled in six DOF while local shaping of the segments is provided by so called warping harnesses. These will correct for focus, astigmatism and trefoil. Hence a mirror with an extreme diameter to thickness ratio of almost 30 is obtained. Its support structure must guarantee a maximum surface form error of $30 \mathrm{~nm}$ rms independent of the segment attitude. Furthermore its stiffness to mass ratio must allow natural frequencies of $50 \mathrm{~Hz}$ or higher to obtain sufficient bandwidth for the actuators that control the piston and tip/tilt of the segment.

Designing such structure is a challenge that has been successfully completed. Three prototypes have been built and are about to be delivered to ESO. This paper discusses the main performance requirements and how they could be transferred into an elegant structure design. Furthermore an overview will be given on the main performance parameters in order to see whether the present design can be further optimized.
\end{abstract}

Keywords: Opto-mechanics, ELT, static determined, M1 support structure.

\section{INTRODUCTION}

The E-ELT is going to be the biggest ground based optical telescope in the world in the coming decades. It will have a hexagonal diameter of $42 \mathrm{~m}$. The primary mirror will consists of 984 hexagonal shaped segments (figure 1) and minimal width of $1.2 \mathrm{~m}$. Because of the hexagonal shape of the segments the mirror can be subdivided in 6 identical sections with 164 different segments (figure 2) due to the parabolic mirror shape. Therefore mass per segments will be different. The challenge has been to design one type of support structure that can be tuned to the specific segment needs.

Each support structure will consist of two main parts being the fixed frame and the moving frame. Due to maintenance of

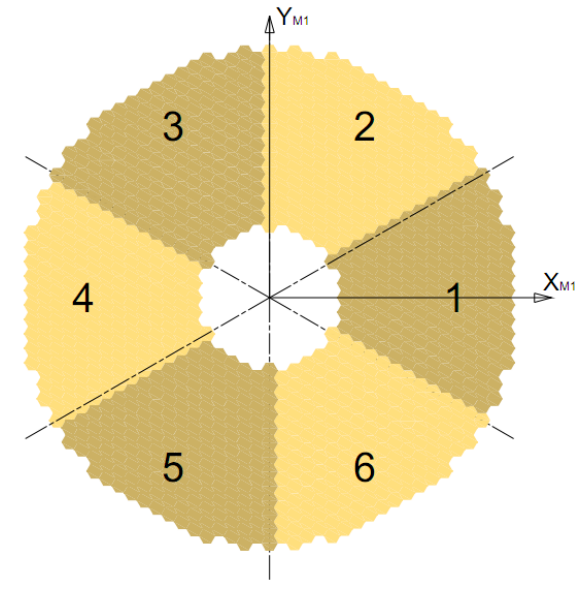

figure 1: M1 segmentation

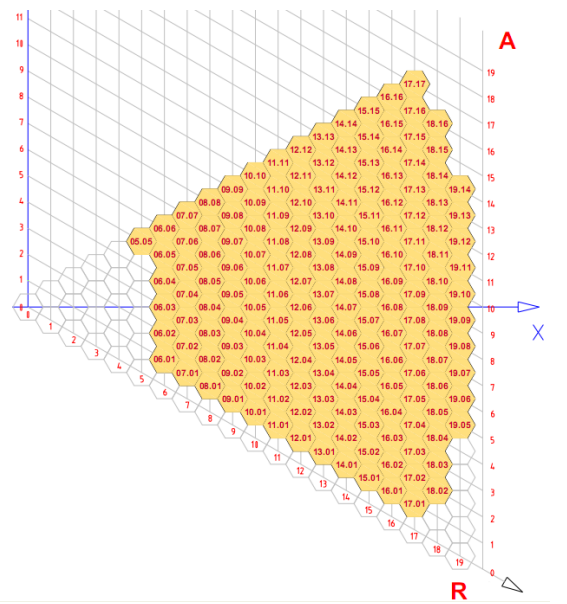

figure 2: Subdivision into 164 hexagonal shaped segment.

Ground-based and Airborne Telescopes III, edited by Larry M. Stepp, Roberto Gilmozzi, Helen J. Hall Proc. of SPIE Vol. $7733,77332 \mathrm{H} \cdot$ (C) 2010 SPIE · CCC code: 0277-786X/10/\$18 - doi: 10.1117/12.857175 
the segments they have to be removed together with the moving frame at regular intervals for mirror recoating. The fixed frame will remain in position. Because recoating takes more time then available during one day, the segment and its support structure is replaced by an identical one. Accessibility of the individual segments will be limited when they are replaced meaning that engagement between moving frame and fixed frame must be flawless.

\section{DESIGN DESCRIPTION}

\subsection{The axial mirror segment support}

A mirror segment is supported at 27 support points such that segment deformation due to gravity is minimized. These points are grouped into 9 tripods with 3 support points each. Three tripods are again supported by another tripod that is hanging down from the moving frame. This is illustrated in figure 3. Such support structure is static determined which means that the support reaction loads depend on the whiffletree geometry and not on its stiffness. An important issue is that the Center of Gravity (COG) of each tripod must coincide with the support strut centerline and its lateral support plane. The six upper tripods carry lower loads and are smaller than the lower tripods.

Because the mirror segment is in fact support at three point perpendicular to its surface three Degrees Of Freedom (DOF) are controlled. These are piston and tip/tilt.

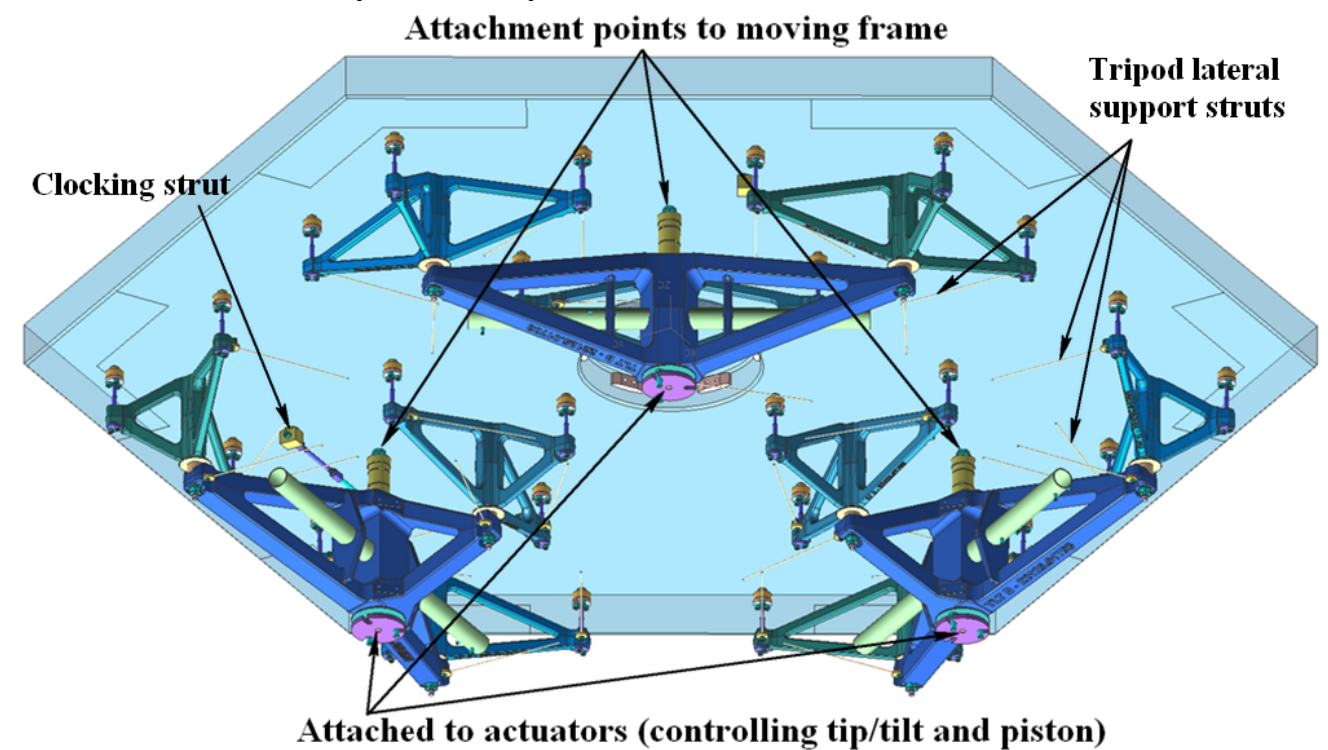

figure 3: The whiffletree support structure of a mirror segment.

\subsection{The lateral mirror segment support}

In order to control the other three DOF of the mirror segment as well (translation in X-and Y-direction together with rotation around the $\mathrm{Z}$-axis) a membrane is installed in the center of the segment (figure 4). Its center is fixed to the moving frame. To enhance its torsion load capability a clocking strut is added at the edge of the segment and also connected to the moving frame. There are three attachment points for the clocking strut. Only one is used which is the one that results in a clocking strut that is parallel to the elevation axis of the telescope. This is to avoid a tension/compression load in this strut when tilting the telescope. This would result in a small rotation of the segment due to its eccentric position.

The tripods also have 6 DOF that need to be controlled. Piston and tip/tilt are controlled through the attached segment support struts and the mirror segment itself. The lateral displacements and clocking motion are controlled by use of three 
co-planar struts that are connected to the end of the tripod sections. At the other end these struts (figure 3) are also connected to the moving frame.

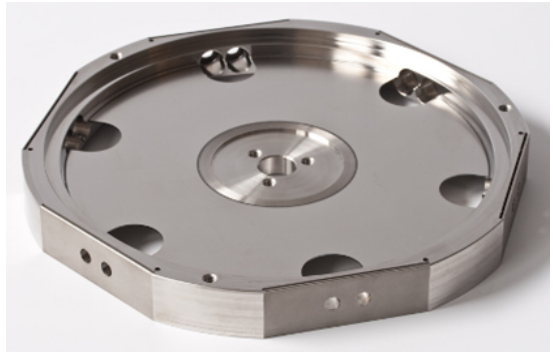

figure 4: Segment membrane

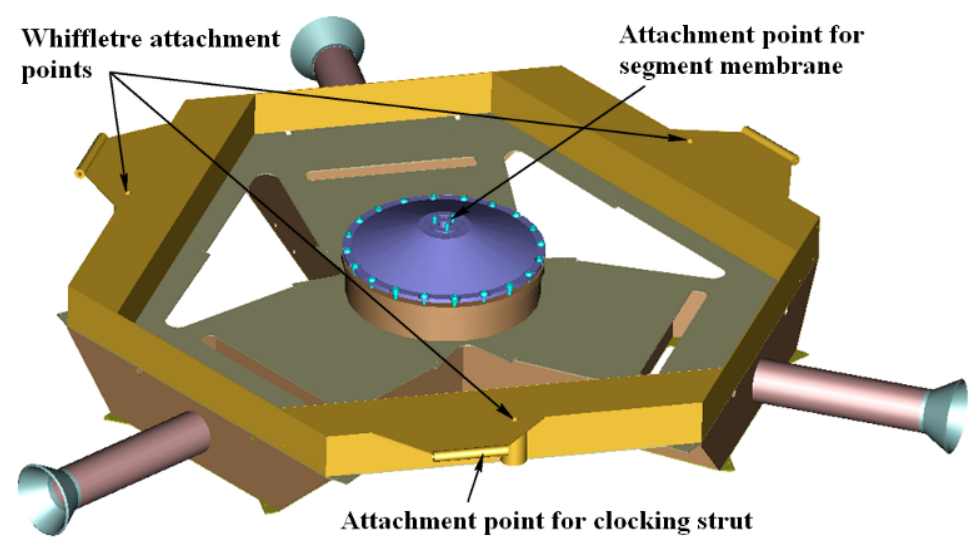

figure 5: The moving frame.

\subsection{Moving frame support.}

From the previous description it can be concluded that the moving frame fully supports the mirror segment. It is connected to "earth" using three actuators that control piston and tip/tilt. The other three DOF are controlled by three moving frame flexures (figure 6 and figure 7). The flexures and actuators are all connected to the fixed frame which is bolted on top of the M1 structural altitude structure. In this way a fully statically determined segment support structure is obtained (figure 8).

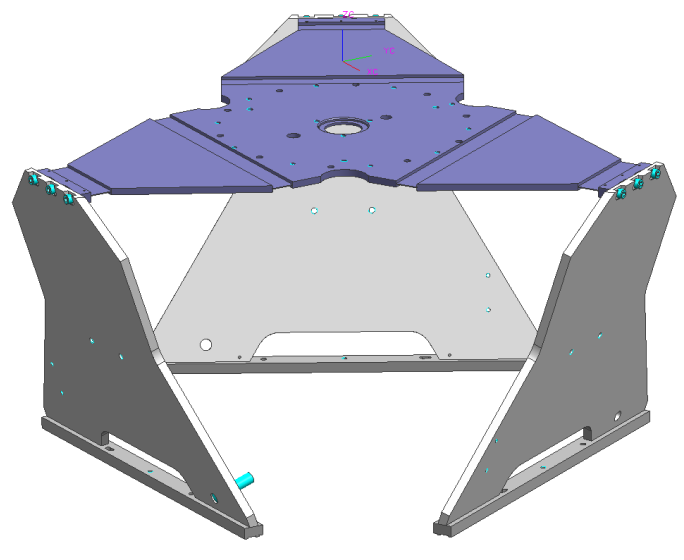

figure 6: Moving frame flexures .

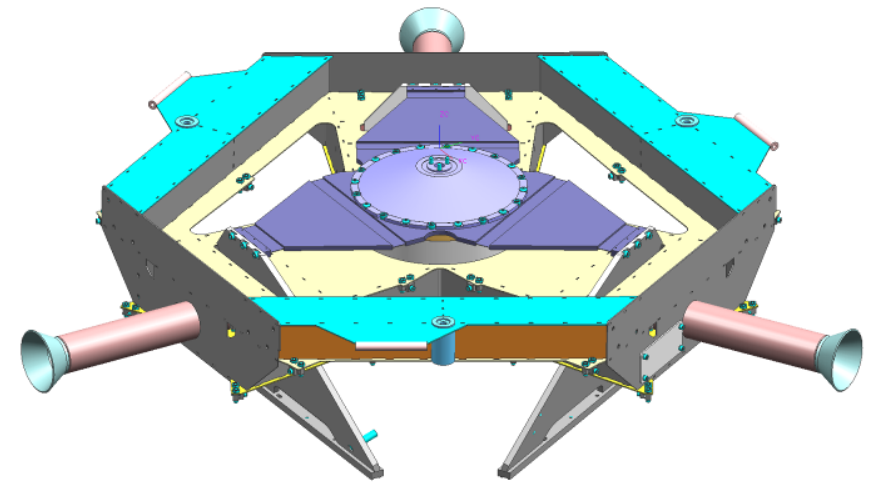

figure 7: Folded leaf spring flexures integrated with moving frame.

One might wonder why this moving frame is needed. Its major function is to provide lateral support for the whiffletrees. Loads resulting from the flexure deformations can now be counteracted by the actuators leading to a small internal deformation of the moving frame. This deformation is not transferred to the segment because of the segment membrane. One should realize that the moving frame is not moving relative to the mirror segment.

It should be noticed that the three supporting actuators compensate for the structural deflection variation of the telescope structure when its elevation is changed. This compensation can be a considerable number of millimeters. The moving frame flexures have to deform to the same amount resulting in considerable loads to the moving frame. 


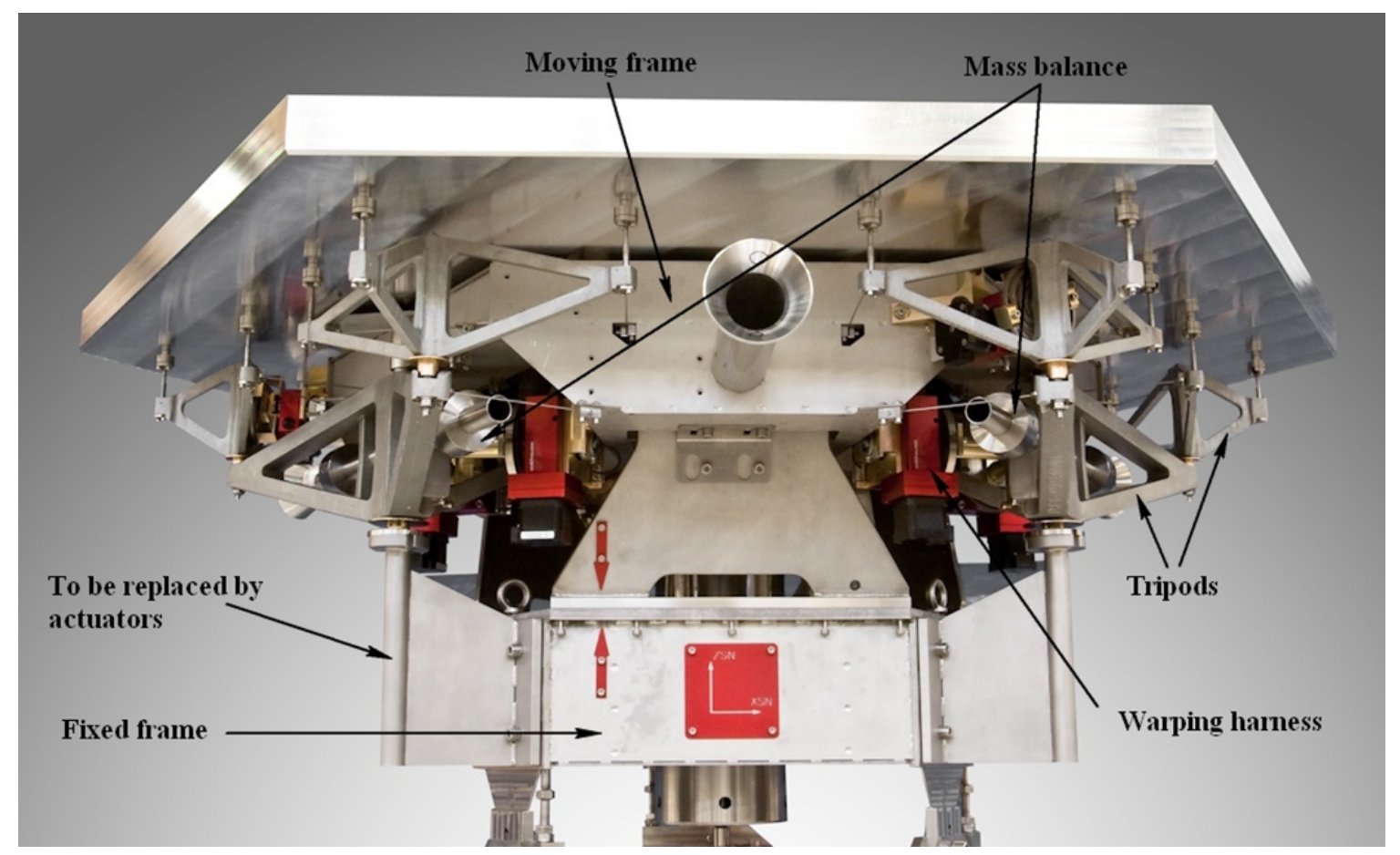

figure 8: The fully assembled support structure of one segment.

\subsection{Passive and active surface control of segments}

As mentioned in the introduction not all segments have an identical shape. Due to the parabolic shape of the primary mirror the segments at the edges of the mirror are few centimeter smaller than the mirror segments near its center . Because all segments have the same support structure it is inevitable that the segment deformations are different and certainly not minimized. The deviation from the ideal segment shape can be calculated for each segment and is decomposed in so called Zernike terms. In doing so it can be shown that the deviations mainly concern defocus, astigmatism and some trefoil. These deformations are compensated by applying bending moments via the whiffletrees to the segment. The moments 2, 4 and 6 compensate for defocus. Moments 1,3 and 5 compensate for astigmatism while moments 7,8 and 9 compensate for trefoil.

Another way of compensating for defocus is by applying mass balance in the center of the segment. It was found that this way a more efficient mass balance is achieved while nearly the same result is obtained. For practical reasons TNO has decided to decompose the applied tripod bending moments as indicated in figure 10.

Two methods exist to apply a moment to the tripod. Either this is done by a mass balance or by an actuator. The first is passive while the other is active. Passive compensation is attractive because it only needs to be done once and does not need sensors, control algorithms etc and is therefore cheap. The nice thing about mass balancing is that the compensating bending moment is dependant on the gravity direction in exactly the same way as the segment gravity deformation itself provided that the mass balance is put at the right place. Putting the mass at same height as the tripod lateral support does this. Each segment will need a different deformation compensating moment. That is achieved by sliding a given mass block along a tube (figure 8). For logistic reasons this is very favorable. 


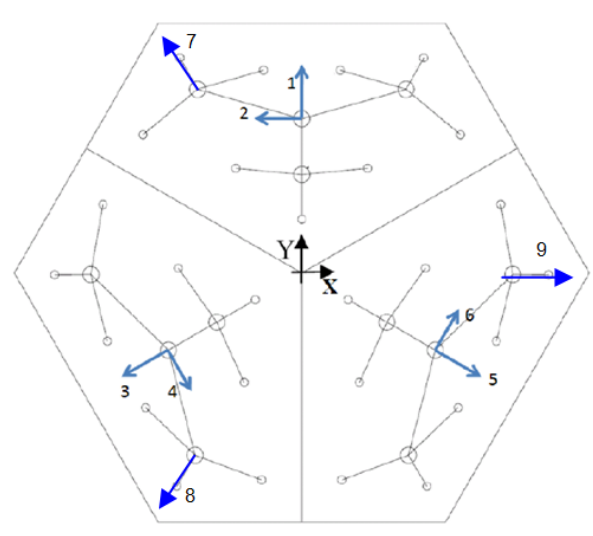

figure 9: Methods of applying moments to the segment to compensate for segment gravity deformations.

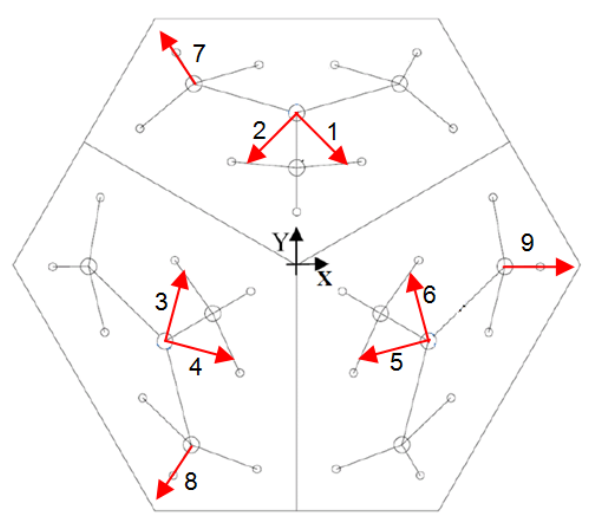

figure 10: Decomposition of applied bending moments.

It has been calculated that the remaining surface form error varies between $8-14 \mathrm{~nm}$ rms. Some typical surface form plots are given in figure 12-figure 13. One can immediately identify the support positions of the segment from this figure.
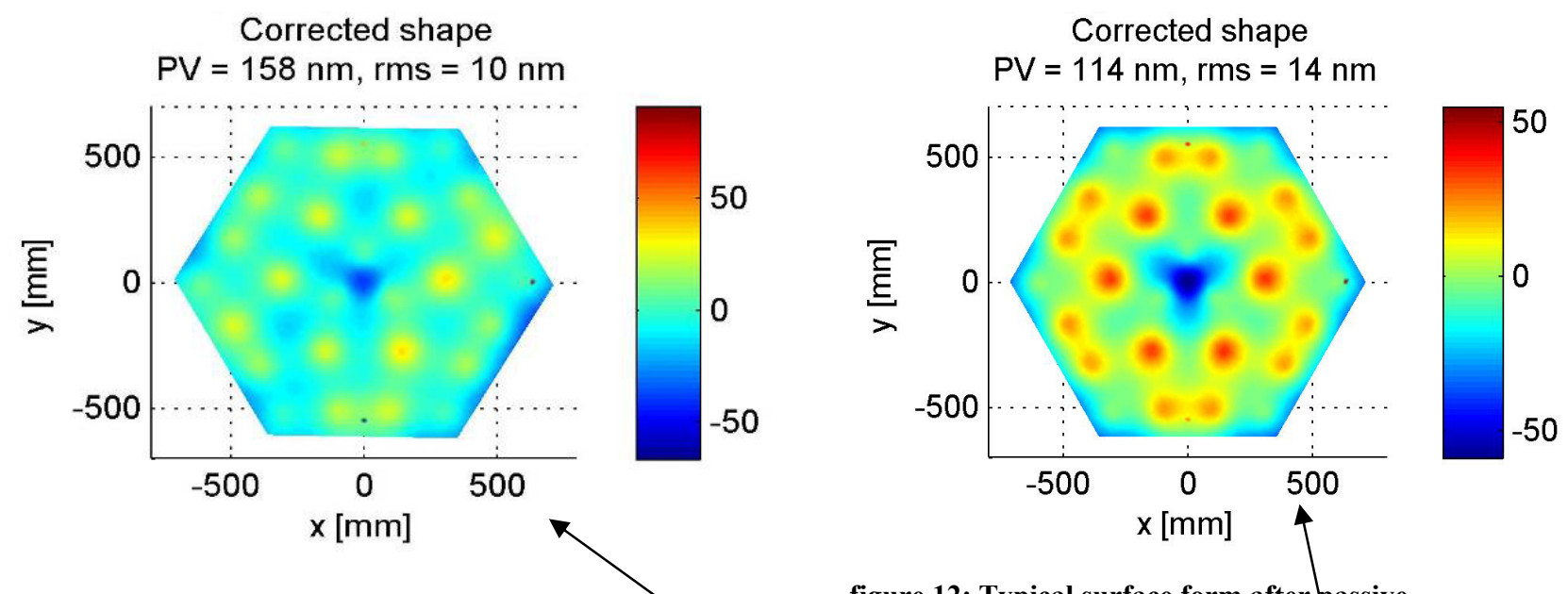

figure 11: Typical surface form after passive compensation of

figure 12: Typical surface form after passive surface form error (segment $@ 0^{\circ}$ ).

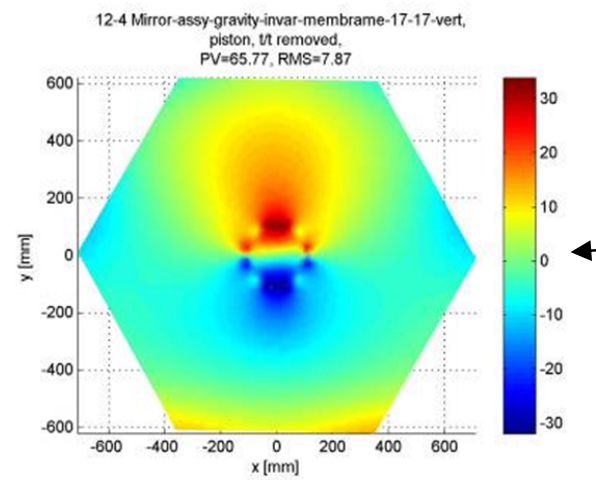

figure 13: Typical surface form deformation (segment @ $90^{\circ}$ )

compensation of surface form error (segment @ $\left.0^{\circ}\right)$..
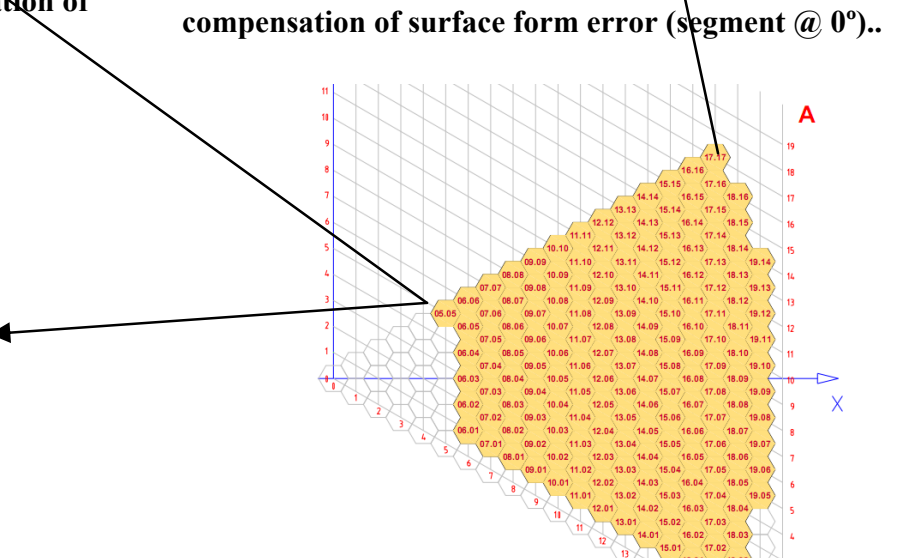
Obviously other sources of surface form deformation exist. E.g. temperature changes cause differences in thermal expansion of the segment and its support structure. This forces the segment support struts to bend in the lateral direction thereby creating a small bending moment. All these moments have the same orientation relative to the segment center thereby creating a defocus error. By careful mechanical design the summation of this error and others like tolerance induced deformations could be kept below $20 \mathrm{~nm}$ rms total.

All mirror segments will be slightly different after polishing is finished. Therefore active control of the segment deformations is also implemented by means of warping harnesses. A stepper motor with self locking gearbox (allows to switch off the motor when no adjustments are needed) is used to rotate a spring thereby creating a bending moment to the tripod to correct for remaining surface form errors after a segment is polished. The coupling between spring and tripod is such that only the bending moment is transferred. Parasitic loads or moments due to e.g. tolerances on positioning of the warping harnesses cause surface form deformations below $1 \mathrm{~nm}$ rms and are therefore negligible.

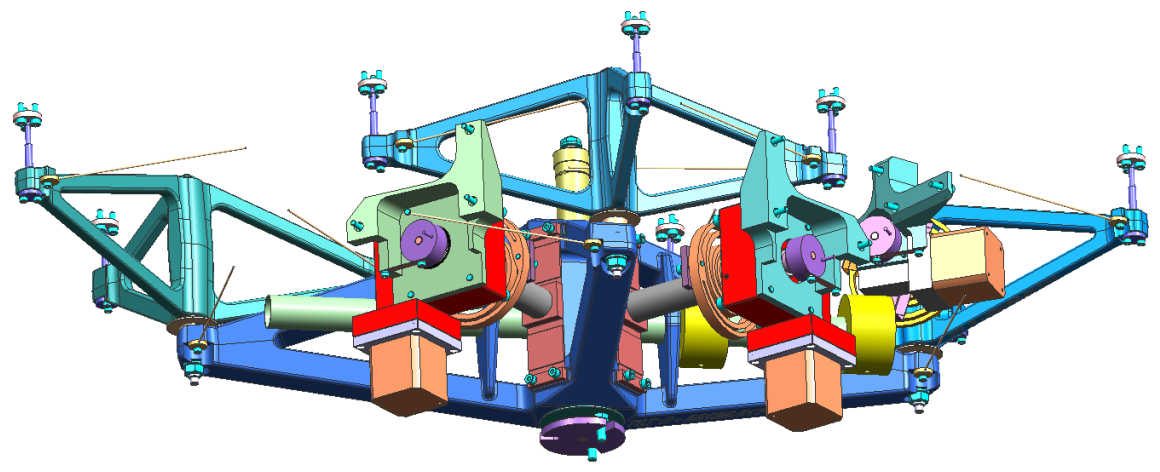

figure 14: Warping harnesses attached to the tripods.

\section{DYNAMICAL PERFORMANCE.}

One of the key performance parameters is the dynamic behavior of the support structure. Each segment has to be kept in position within a few nanometers by the three actuators. To be able to do so these actuators need sufficient bandwidth to counteract the external disturbances of the segment position. These are e.g. wind loads and ground vibrations. Therefore the natural frequencies need to be sufficiently high otherwise controlling the segment surface might prove to be very difficult. The design as made by TNO meets or surpasses the requirements. The obtained frequencies are:

table 1: Lowest natural frequencies of support structure.

\begin{tabular}{|l|c|}
\hline Eigen mode shape & Obtained frequency \\
\hline Clocking & $29 \mathrm{~Hz}$ \\
\hline Tip/tilt & $49 \mathrm{~Hz}$ \\
\hline Translation in X- and Y-direction & $56 \mathrm{~Hz}$ \\
\hline Translation in Z-direction & $62 \mathrm{~Hz}$ \\
\hline
\end{tabular}

Obtaining the required clocking frequency appeared to be difficult. The central membrane, mounted in the center of the segment, needs to be thin to avoid loads perpendicular to the segment due to e.g. tolerances, flexure deformation etc. Tolerances as small as $0.2 \mathrm{~mm}$ are big enough to cause serious surface form deformations. Tilting of the telescope causes a variable deformation of the whiffletrees for which the membrane also has to compensate. A thin membrane however is not capable to provide sufficient clocking stiffness to the segment that weighs around $167 \mathrm{~kg}$. That is why an additional strut has been attached to the segment in the tangential direction. At the other end it is fixed to the moving frame. The outstanding stiffness properties of the moving frame makes the achieved clocking frequency possible. 
The piston and tip/tilt frequencies are determined by the stiffness of the whiffletrees. The fact that the whiffletrees are static determined makes it possible to define a simple analytical model of the whiffletree that allows optimization for stiffness. That has been done resulting in a whiffletrees design where all elements contribute to the same amount in the total whiffletree stiffness. The mathematical model is such accurate that it predicts $66 \mathrm{~Hz}$ as piston frequency (figure 15). Similarly also the tip/tilt frequency could be predicted to be $52 \mathrm{~Hz}$.

Obtaining high natural frequencies is always a matter of realizing highest stiffness at minimum mass. In case of the whiffletrees this has been realized in the most optimal way. All sections of the tripods are loaded in tension or compression meaning that the full cross section has identical stress and contributes optimal to stiffness. The same applies to the struts of the whiffletrees. This has resulted in a mass for the whiffletrees of only $16 \mathrm{~kg}$ total.

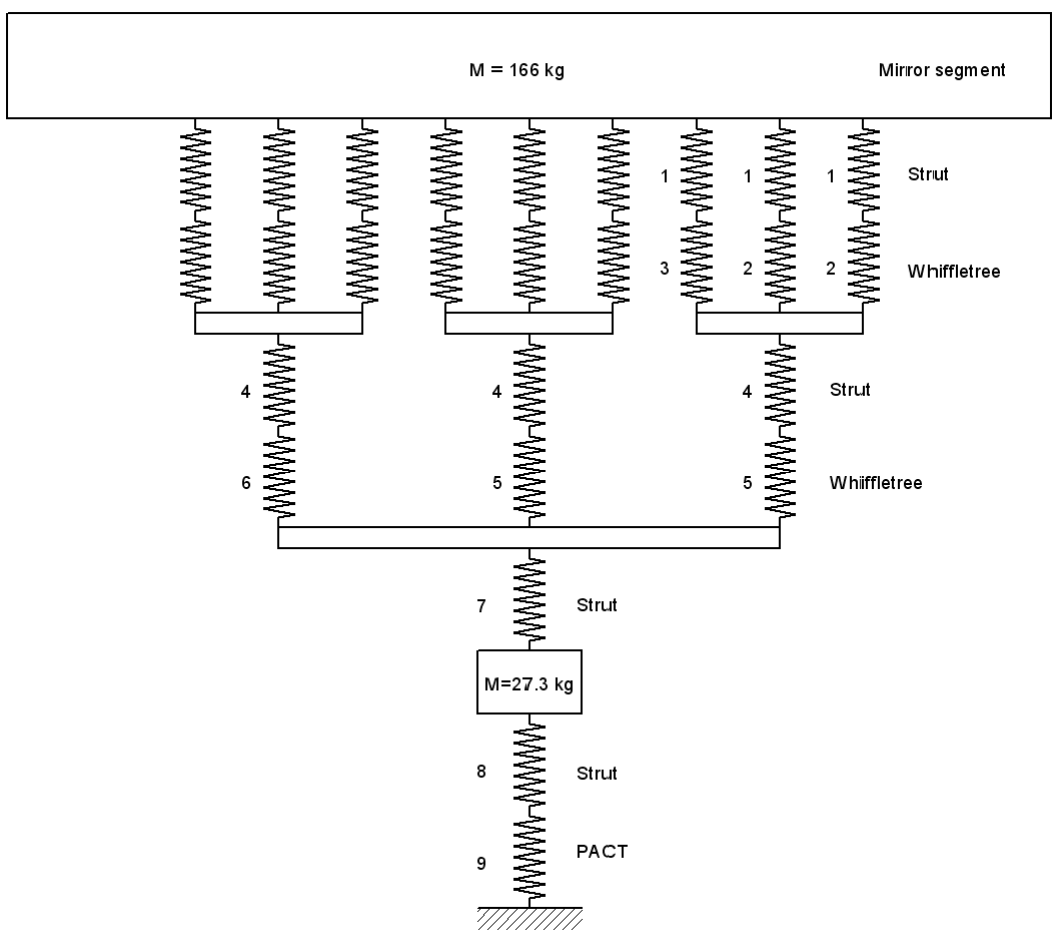

figure 15: Reduced spring model of whiffletree (only one shown).

The moving frame flexures (figure 6) provide the required stiffness for clocking and lateral stiffness for the segment. These structural elements are loaded in shear. This means also that the full cross section of these flexures contribute equally to the stiffness. Also here an optimal mass to stiffness ratio has been achieved. The mass of the flexures amounts to $19 \mathrm{~kg}$. The mass of the fixed frame is relatively high $(48 \mathrm{~kg})$ due to its relative small foot print and the presence of the extractor (lifting device for segment sub assembly). The total mass of the full segment support structure including nine warping harnesses, cabling etc amounts $126 \mathrm{~kg}$ bringing the total mass of one segment with support structure at $293 \mathrm{~kg}$. A mass of max $10 \mathrm{~kg}$ is taken for one actuator

\section{THE ECONOMIC ASPECT.}

One of the challenges for the mechanical design of the support structure was to realize a structure that is very cost effective. A total of 1148 support structures will have to be made which means that production methods had to be chosen that are compliant to series production. The moving frame and fixed frames are both made of sheet metal parts obtained through laser cutting. Assembly of these parts is done by welding. The assembled parts are such that no jigs are needed for welding thereby realizing another important cost reduction. The tripods of the whiffletrees are aluminum castings. This proves to be very cost effective. Even for the

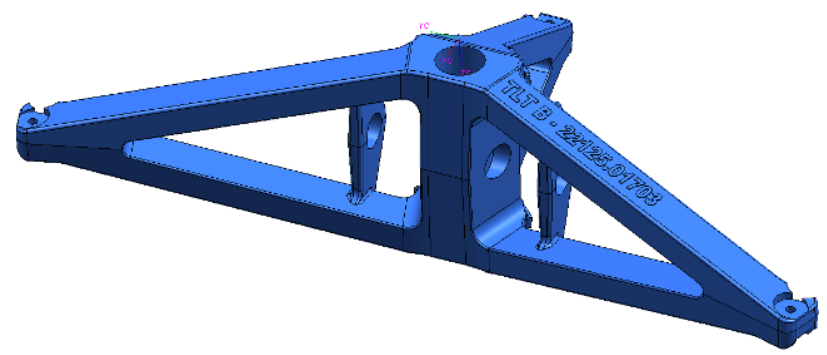

figure 16: Typical example of casted tripod. 
prototypes that were build it was found to be cheaper than production methods like machining or welding. Aluminum was chosen instead of steel because steel tripods would have much thinner tripod cross sections which has a very negative impact on the casting quality.

The warping harness mainly consists of off-the-shelf items. Only its mounting bracket and the coupling to the tripods had to be designed and specifically made. A set of torsion springs can be manufactured in one operation because of its prismatic shape. The machine that manufactures them operates fully automatically in continuous operation during the night. Hence labor hours are minimized.

\section{SENSITIVITY FOR TOLERANCES.}

Due to mechanical tolerances none of the support structures will be fully identical. This is expressed in differences in dimensions, positions and the mass distributions of the whiffletrees. They will influence the segment surface form deformation due to gravity. Dimensional differences of the whiffletree parts itself and its environment cause that its geometry varies thereby providing a slightly varying support stiffness distribution to the segment. Another effect is that the elastic hinges get slightly deformed thereby introducing bending moments that will influence the segment shape. Mass variations of e.g. the tripods have a similar effect. It was found that the castings are very repeatable in size and mass distribution. The resulting surface form deviations are that small that they proved to be acceptable without further compensation. That means that there is no need to mass balance the individual tripods which is a major asset of the design as it stands.

Dimensional differences in the moving frame might cause a lateral displacement of the tripods. That is effectively counteracted by making the lateral support struts of the tripods adjustable in length. Having produced three prototypes it can now be concluded that production is that well repeatable that the length of the struts can be fixed because the measured tolerances are only few tenths of a millimeter.

\section{EXCHANGE OF SEGMENT ASSEMBLY.}

With 984 segments installed in the E-ELT it will be necessary to replace one or two segments every day for recoating. This way each segment will be recoated within 2 years. Because this will be a frequent operation it is obvious that replacing a segment should be done quickly, without too much knowledge and skills of the operator and without realignment. At the prototypes it was found that removing a segment assembly can be done within 10 minutes. The same applies to remounting. The mounting reproducibility is around $10 \mu \mathrm{m}$ thereby proving that remounting can be done without realignment.

To lift the segment assembly from its fixed frame an extractor is included in every fixed frame. In preparation of lifting the segment assembly the extractor is extended to engage with the segment assembly. Then the bolt connections between segment

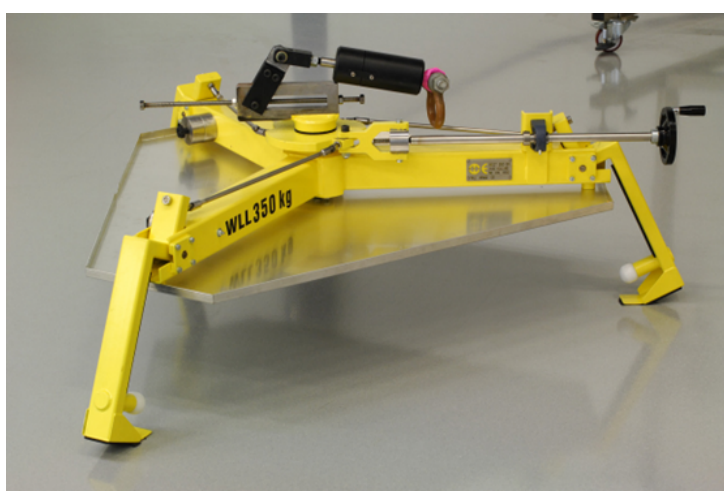

figure 17: Segment manipulator prototype (manual version). assembly and fixed frame are removed allowing the operator to lift the segment assembly. At its highest position a segment manipulator (presently manually operated but intended to be fully controlled in 6 DOF in future) will take over the segment assembly and the extractor can be disconnected and retracted.

The segment installation is done in reversed order. Alignment features on the extractor will guarantee that the angular positioning of the segment assembly is automatically brought within the capture range of the alignment provisions on the fixed frame. 


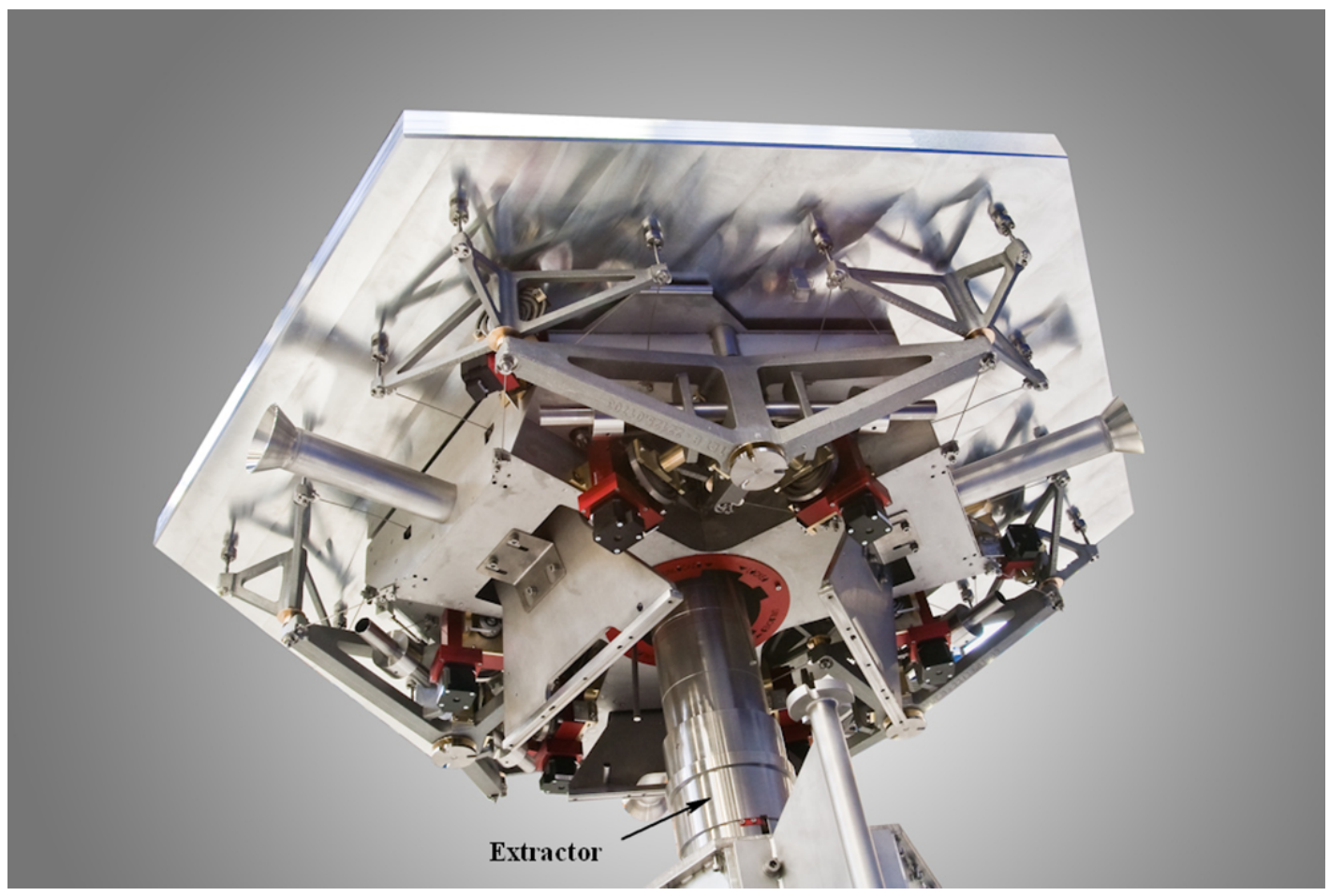

figure 18: Segment sub-assembly and fixed frame disconnected.

\section{FINAL REMARKS AND CONCLUSIONS}

Having described the main aspects of the support structure of the M1-segments it can be concluded that:

1. The performance of the segment support structure depend to a large extend on the fact that it is static detemined and highly decoupled i.e. the parasitic stiffness is very low.

2. The connection the between segment subassembly and the fixed frame is static determined and guarantees therefore the interchangeability of the segment subassemblies.

3. The remounting accuracy of the segment subassembly is better than $10 \mu \mathrm{m}$.

4. The time needed for dismounting/remounting of a segment subassembly is less than 10 minutes. 